DePauw University

Scholarly and Creative Work from DePauw University

$1-2020$

\title{
The role of gender in the relations among Dark Triad and psychopathy, sociosexuality, and moral judgments
}

Kevin E. Moore

DePauw University, kemoore@depauw.edu

Scott R. Ross

DePauw University, srross@depauw.edu

Evelyn C. Brosius

DePauw University

Follow this and additional works at: https://scholarship.depauw.edu/psyc_facpubs

Part of the Psychology Commons

\section{Recommended Citation}

Moore, K. E., Ross, S. R., \& Brosius, E. C. (2020). The role of gender in the relations among Dark Triad and psychopathy, sociosexuality, and moral judgments. Personality and Individual Differences, 152, 109577. DOI: https://doi.org/10.1016/j.paid.2019.109577.

This Article is brought to you for free and open access by the Psychology and Neuroscience at Scholarly and Creative Work from DePauw University. It has been accepted for inclusion in Psychology and Neuroscience Faculty Publications by an authorized administrator of Scholarly and Creative Work from DePauw University. 
The Role of Gender in the Relations Among Dark Triad and Psychopathy, Sociosexuality, and Moral Judgments

\author{
Kevin E. Moore \\ Scott R. Ross \\ Evelyn C. Brosius \\ Department of Psychology and Neuroscience \\ DePauw University \\ 7 East Larabee Street \\ Greencastle, IN 46135 \\ USA
}

Corresponding author: Kevin Moore kemoore@depauw.edu 


\begin{abstract}
The Dark Triad (D3) traits of narcissism, Machiavellianism, and psychopathy have been linked to a range of moral behavior, and to sociosexuality and sexual behavior, particularly in males. The current study examined whether males and females differ with respect to relations among D3 traits and dimensions of psychopathy from the Triarchic Psychopathy Measure (TriPM), sexual behavior, sociosexuality, tendency towards infidelity, and moral judgments, using a community sample. D3 and TriPM measures were generally more strongly correlated with sexual behavior for males in ways that might increase numbers of matings and sexual partners, but were more strongly correlated with intention towards infidelity and sociosexual attitudes for females - in ways that might facilitate extra-pair mating. Sociosexuality and self-reported sexual behavior showed a strong pattern of overall and gender-specific relations to moral judgments. D3 and psychopathy measures were also related to everyday moral judgments, although generally less so than were sociosexuality and sexual behavior. These results clearly point to the importance of taking gender into account when considering the relations between D3 traits, sexual behavior, sociosexuality, and moral judgments.
\end{abstract}

Keywords: Psychopathy, Sociosexuality, Gender, Morality, Dark Triad, Sexual Behavior, Infidelity 


\section{Declaration of Interest}

None 


\section{Introduction}

The Dark Triad (D3) of personality (Paulhus \& Williams, 2002) represents a constellation of Machiavellianism, narcissism, and psychopathy. These traits are associated with a host of maladaptive outcomes, including sex-related issues, interpersonal difficulties, and atypical moral judgments (Muris, Merckelbach, Otgaar, \& Meijer, 2017). Psychopathy has been associated with impaired social relationships and social contract reasoning (Ermer \& Kiehl, 2010). The D3 has also been associated with altered decision-making, especially in men (Karandikar, Kapoor, Fernandes, \& Jonason, 2018), less moral concern for harm (Jonason, Strosser, Kroll, Duineveld, \& Baruffi, 2015), and is generally believed to reflect a largely immoral or amoral conscience (e.g., Hare, 1999).

Although traditionally viewed as maladaptive and pathological, recent evidence suggests that D3 traits may have adaptive components in some domains. D3 traits may be associated with increased short-term mating success in males (Jonason, Li, Webster, \& Schmitt, 2009).

Holtzman and Strube (2013) found that D3 traits were correlated with self-adornment to increase attractiveness, and Carter, Campbell, and Muncer (2014a) found that men high in D3 traits were more attractive to women. Such studies point to a variety of outcomes associated with D3 traits, and other research suggests that the outcomes might differ by gender. For example, women's moral judgments about intimate and sexual behavior violations appear harsher than men's, and sociosexuality influences those judgments (Selterman \& Koleva, 2015). In non-mating domains gender appears to moderate the relation between psychopathy and two very widely accepted behavioral markers of the disorder, response perseveration and passive avoidance (see Efferson \& Glenn, 2018, for a review). 
The existing evidence shows that D3 traits and psychopathy are linked to human mating and morality, and that these associations may differ by gender. Past studies on psychopathy have often not taken gender into account, or have identified possible functional impacts of D3 traits on male mating. It is, however, possible that D3 traits may have functional impacts that manifest differently in males and females. The nascent state of research on how D3 traits may function differently in males and females does not allow for clear a priori hypotheses, but highlights the importance of beginning to explore how gender may impact the pattern of association of D3 traits with mating behavior, sociosexuality, and moral judgments.

\subsection{Current Study}

We examined the role of gender in relations among D3 traits and psychopathy, sexual behavior/sociosexuality, and moral judgments, using the Short Dark Triad (SD3; Jones \& Paulhus, 2014), the Triarchic Psychopathy Measure (TriPM; Patrick, Fowles, \& Krueger, 2009), the Morality of Everyday Life Scales (MELS; Lovett, Jordan, \& Wiltermuth, 2012), the Sociosexual Orientation Inventory - Revised (SOI-R; Penke \& Asendorpf, 2008), items reflecting sexual behavior, and the Intentions Towards Infidelity Scale (Jones, Olderbak, \& Figueredo, 2011).

\section{Methods}

\subsection{Participants and Procedure}

272 participants (127 female, 145 male) who were 18 years of age or older completed an anonymous survey via Amazon Mechanical Turk. Participants received \$2.25 for taking part. The research was approved by the appropriate university Institutional Review Board. Participants read and responded to consent information before completing the on-line anonymous questionnaire. 


\subsection{Measures}

The 27-item SD3 (Jones \& Paulhus, 2014) assesses narcissism, Machiavellianism, and psychopathy. Higher scores indicate higher levels of the traits. The 58-item TriPM (Patrick et al., 2009) assesses psychopathy along three dimensions-boldness, meanness, and disinhibition. Higher scores indicate higher levels of psychopathy. The 9-item SOI-R (Penke \& Asendorpf, 2008) assesses tendency toward longer- or shorter-term mating orientation, and yields a score for past behaviors linked to mating orientation, attitudes concerning mating orientation, and desire, as well as an overall score. Higher scores indicate more short-term (less committed) mating orientation. The 7-item ITIS (Jones et al., 2011) attempts to assess intention to commit infidelity. Higher scores indicate a greater predisposition to infidelity. The 30 -item MELS (Lovett et al., 2012) assesses judgments of wrongfulness of relatively typical moral violations/actions. The MELS yields a total score, as well as separate scores for deception, harm (to an in-group member), laziness (acting in a wrongful way due to convenience), failure (to act morally), body (e.g., body "violations" including some types of sexual behavior), and disgust (e.g., poor hygiene). Lower scores indicate lower wrongfulness judgments. Participants also responded to items that asked about sex/gender, number of past sexual partners, age at first sex, ideal lifetime sexual partner number, preference for multiple versus individual relationship partners (5-point scale, $1=$ strong preference for multiple simultaneous partners, $5=$ strong preference for single partner at a given time), and other demographic variables.

\section{Results}

We calculated Pearson bivariate correlations between measures. We examined whether correlations between measures differed by gender using multiple indices: (1) noting whether the 
correlation was significant only for one gender; (2) t-tests for differences in correlations; (3) whether gender significantly moderated relations between variables.

\subsection{D3 and Psychopathy Measures and Sexual Behavior, Sociosexuality, \& Intentions Toward} Infidelity.

Table 1 shows the correlations by gender for D3 and TriPM measures with sexual behavior, SOI, and ITIS scores. As shown in Table 1, 74 of 126 possible correlations (58.7\%) were statistically significant, with 41 of $63(65.1 \%)$ possible correlations significant for males, and 33 of 63 (52.3\%) correlations significant for females. In 31 of 63 cases $(49.2 \%)$, the correlations were either significant only for one gender, differed significantly from one another, or showed a significant moderating effect of gender. We describe this pattern below.

\subsubsection{D3 Components, Psychopathy, and Sexual Behavior, Sociosexuality, and Intentions}

\section{Towards Infidelity}

D3 Machiavellianism was significantly positively correlated to number of past sexual partners only for men. Machiavellianism was significantly positively correlated with SOI-R Attitude only for women. Machiavellianism was significantly positively related to SOI-R Desire and SOI-R Total scores in a similar manner for both genders. Finally, Machiavellianism significantly predicted ITIS score for both genders, but this relation was stronger for women (i.e., gender moderated the correlation).

D3 Narcissism showed a strong pattern of relations with measures of sexual behavior, SOI, and infidelity intention, and these relations often differed by gender. Narcissism was significantly positively related to ideal partner number and to past partner number only for men. Narcissism was significantly positively correlated to SOI-R Behavior only for men. In contrast, 
Narcissism was significantly positively related to SOI-R Attitude, Desire, and Total only for women. Narcissism was strongly positively correlated with infidelity scores for both genders. D3 Psychopathy and TriPM Total scores were closely correlated $(r=.73)$, and both measures were related to nearly all sexual and sociosexuality measures. The pattern of relations, however, differed by gender. Both D3 Psychopathy and TriPM Total were significantly predictive of higher numbers of past partners, ideal partners, and age at first sex, and the relation was stronger for men than for women. D3 Psychopathy was more strongly predictive of preference for multiple partners in men, whereas the significant relation between TriPM Total and multiple-partner preference did not differ by gender. D3 Psychopathy was significantly positively related to SOI-R Behavior for both men and women, but this relation was stronger in males. TriPM Total was also significantly correlated with SOI-R Behavior in both genders; the correlation was higher for males, but not significantly. D3 Psychopathy was significantly positively related to SOI-R Attitude, Desire, and Total scores in both males and females. TriPM Total was significantly positively correlated with SOI-R Attitude and Total in both genders, and SOI-R Desire for only women. Both psychopathy measures were significantly predictive of ITIS scores for both men and women, and the correlations were especially strong for women. The correlation between D3 Psychopathy and infidelity intention was higher for women than for men, but the difference was not significant. The correlation between TriPM total score and intention toward infidelity was significantly higher for women than for men.

\subsubsection{Psychopathy Dimensions and Sexual Behavior, Sociosexuality, and Intentions Towards}

\section{Infidelity}

Finding such strong gender differences for psychopathy, we next sought to determine which dimensions of psychopathy seem to be driving these differences in sociosexuality/sexual 
behavior. TriPM Boldness was positively correlated with past partner number only for men. Boldness was correlated with multiple relationship partner preference only in women. Boldness was positively related to SOI-R Behavior in both genders, but positively related to SOI-R Attitude and Total only for women. Notably, TriPM Boldness was unrelated to infidelity intention in both genders.

In contrast, TriPM Meanness was positively correlated with ideal and past partner number only for men. Similarly, Meanness was positively related to multiple partner preference and SOI-R Behavior only for men. Meanness was positively correlated with SOI-R Attitude, Desire, and Total scores for both genders. Meanness was strongly positively related to infidelity intention for both genders, but this relation was moderated by gender, and stronger for women $(r=.556)$ than men $(r=.417)$.

TriPM Disinhibition also demonstrated effects stronger than Boldness. Disinhibition was positively related to ideal partner number and multiple partner preference only in men. Disinhibition was positively correlated with SOI-R Behavior, Desire, and Total in both genders, but was significantly positively correlated with SOI-R Attitude only in women. Disinhibition was strongly and significantly correlated with infidelity intention in both genders, but the relation was moderated by gender, and the correlation was significantly higher for women $(r=.564)$ than for men $(r=.372)$.

\subsection{Sexual Behavior/Attitudes, Sociosexuality, Intentions Towards Infidelity, and Everyday}

\section{Moral Judgments}

Table 2 shows how measures of sexual behavior/attitudes, sociosexuality, and infidelity are associated with MELS subscales and total. As Table 2 shows, 65 of 126 (51.8\%) possible correlations were significant; 36 of $63(57.1 \%)$ were significant for men, while 29 of 63 (46\%) 
were significant for women. Measures of sexual behavior/attitudes, sociosexuality, and infidelity were related to moral judgments, and the pattern was different for men than for women. The correlation was either significant only for one gender, differed significantly between genders, or showed a significant moderating effect of gender in 25 of $63(39.7 \%)$ cases. We discuss this pattern below.

\subsubsection{Measures of Sexual Behavior and Everyday Moral Judgments}

Ideal partner number was significantly negatively correlated to judgments of the moral wrongness of Deception and Harm only for men. In contrast, ideal partner number was significantly inversely related to moral wrongfulness judgments on the Lazy, Body, and Disgust MELS subscales only for women. Ideal partner number was negatively related to MELS Total score for both men and women, but gender significantly moderated the relation.

The number of past partners was significantly negatively correlated with judgments of the moral wrongfulness on the MELS Total, and on the Deception and Harm dimensions for men. The number of past partners was unrelated to any MELS score in women. Age at first sex was positively correlated to wrongfulness judgments on MELS Harm, Laziness, Failure, Disgust, and Total, but only in men. Age at first sex was positively related to views of moral wrongfulness on the Body dimension of the MELS for both genders. Multiple partner preference was significantly related to perceived wrongfulness on the Deception and Harm dimensions of the MELS only for men.

\subsubsection{Sociosexuality (SOI-R) and Everyday Moral Judgments}

SOI-R Behavior score was significantly and inversely related to moral wrongness judgments on the MELS Deception dimension only for women. The reverse was true for the judgments on the Harm dimension-SOI-R Behavior scores were inversely related to Harm 
judgments only for men. SOI-R Behavior was significantly and negatively related to moral judgments on the Body dimension and MELS Total score for both women and men.

SOI-R Attitude scores were significantly and negatively related to the moral wrongness of Deception only for women. SOI-R Attitude scores were significantly negatively correlated with judged moral wrongness on Laziness, Failure, Body, and Total MELS score for both genders. SOI-R Attitude was negatively related to perceived moral wrongfulness on the Disgust dimension only for women.

SOI-R Desire was significantly associated with reduced judgments of wrongfulness on the Deception, Body, Disgust, and MELS Total dimensions for both men and women. SOI-R Desire was significantly linked with reduced judgments of wrongness on the Harm dimension only for females. In contrast, SOI-R Desire was linked to reduced wrongfulness judgments on the Laziness and Failure dimensions of the MELS only for men.

SOI-R Total scores were significantly associated with judgments of lower wrongfulness on MELS Deception for both men and women, but gender moderated the relation, indicating a stronger effect in women. SOI-R Total was significantly related to judgments of lower wrongfulness on the Disgust dimension only for women. SOI-R Total score was correlated with judgments of lower wrongfulness in both women and men for MELS Harm, Laziness, Failure, Body, and Total scores.

\subsubsection{ITIS and Everyday Moral Judgments}

Infidelity intention, as measured by the ITIS, was significantly linked to lower wrongfulness judgments on the Deception and Harm dimensions, and to increased judgments of wrongfulness on the Laziness dimension, for both genders.

\subsection{D3 and TriPM measures and Everyday Moral Judgments}


Table 3 shows how D3 subscales (Machiavellianism, narcissism, and psychopathy), and TriPM scores are associated with everyday moral judgments (MELS subscales and total). D3 and TriPM measures showed a smaller range of correlations with moral judgments than did the measures of sexual behavior/attitudes and sociosexuality shown in Table 2. As Table 3 illustrates, 35 of $98(35.7 \%)$ possible correlations were significant; 20 of 49 (40.8\%) were significant for men and 15 of $49(30.6 \%)$ were significant for women. The pattern of associations shown in Table 3 is somewhat different for men than for women. The correlation was either significant only for one gender, differed significantly between genders, or showed a significant moderating effect of gender in 7 of $49(14.3 \%)$ cases. We discuss this pattern below for each set of relations.

\subsubsection{D3 Measures and Everyday Moral Judgments}

D3 Machiavellianism was significantly correlated with reduced judgments of the moral wrongfulness of MELS Deception for both men and women. Machiavellianism was correlated with judgments of greater wrongfulness on MELS Disgust only for men. D3 Narcissism was significantly related to reduced wrongfulness judgments on MELS Deception, and to increased moral condemnation of the MELS Laziness dimensions, in both genders. Narcissism was linked to judgments of greater wrongfulness of MELS Disgust only in men. D3 Psychopathy scores were significantly related to decreased judgments of wrongfulness on MELS Deception and Harm, and increased wrongfulness judgment of Laziness for both genders. D3 psychopathy was significantly linked to judgments of greater wrongfulness on MELS Disgust only for men.

\subsubsection{TriPM Measures and Everyday Moral Judgments}

TriPM Total score was significantly correlated with judgments of reduced wrongfulness on MELS Deception and Harm dimensions, and with greater wrongfulness of Laziness, for both 
genders. TriPM total score was also significantly linked to greater judged wrongfulness on MELS Disgust, but only for men. Boldness was significantly correlated with judgments of greater wrongfulness on MELS Laziness for women, but not for men. Meanness was significantly correlated with reduced perceived wrongfulness on the MELS Deception and Harm dimensions, and with perceptions of greater wrongfulness of Laziness, for both genders. Meanness was associated with reduced judgment of moral harm of Failure only for men. Disinhibition was associated with reduced perception of the moral wrongness of MELS Deception and Harm in both genders, and was associated with judgment of greater harm of Laziness in only men.

\section{Discussion}

D3 and TriPM measures showed a stronger pattern of correlations with sexual behavior and the SOI-R Behavior scale for males than for females. The results presented in section 3.1 show that 19 of 35 (54.3\%) relations between D3 and TriPM scores and sexual behavior and the SOI-R Behavior index were stronger for men than for women, while only 1 of $35(2.9 \%)$ was stronger for women. This is consistent with past research (e.g., Jonason et al., 2009), and suggests that D3 traits in general, and psychopathy in particular, may facilitate higher frequency of mating and higher numbers of mates for men.

In contrast, psychopathy measures showed a more robust pattern of associations with non-behavioral components of the SOI-R for women than for men. None of the correlations between D3 and TriPM measures with the SOI-R Attitude and Desire scales were higher for men than women, while 6 of $14(42.9 \%)$ were higher for women than for men, and 6 of the remaining 8 were stronger for women (but not significantly so). This pattern is consistent with some of the results reported by Carter, Campbell, and Muncer (2014b), who found a nominally higher 
correlation between D3 traits and some attitudinal measures of mating in women than in men. Our results suggest that while not dramatically increasing numbers of matings, D3 traits and psychopathy increase motivation and openness to short-term mating in women, perhaps facilitating extra-pair mating. This interpretation is bolstered by our finding that D3 and TriPM scores showed a much more robust relation to intention to infidelity for women than for men. As far as we are aware, this is the first demonstration that D3 constructs are more strongly related to infidelity intention in women than in men. None of these correlations were stronger for men than women, whereas 4 of 7 (57.1\%) were stronger for women, and the remaining non-significant differences were in the direction of being stronger for women. This pattern suggests that D3 constructs, including psychopathy, are related to women's mating behavior, but manifest as an increased openness to infidelity, without substantially affecting overall numbers of mates. D3 traits may thus manifest differently by gender in ways that are consistent with male- and femaletypical sexual strategies (Buss \& Schmitt, 1993; Greiling \& Buss, 2000), facilitating higher frequency of mating in males and increased openness to more focused extra-pair mating in females. Of particular interest was that TriPM Meanness and Disinhibition seemed to be driving this relationship between psychopathy and infidelity for both sexes, and especially for females. Meanness has been construed as "coldheartedness" (Hall \& Benning, 2006; Patrick et al., 2009), but in this context may reflect emotional detachment, more than envy or revenge, to facilitate openness to exploitation of extra-pair mating opportunities. Disinhibition might then facilitate engaging in the behavior. Our results point to the importance of considering gender, and looking at a broad range of mating measures in any discussion of the relation between D3 traits, sociosexuality, and human mating. 
As described in sections 3.2 and 3.3, D3 and TriPM scores, and sexual behavior and sociosexuality, were associated with moral judgment patterns. One somewhat surprising aspect of these patterns was that D3 and TriPM measures - both measures of personality one might think are intrinsically linked to morality—-showed a smaller range of correlations with moral judgments than did the measures of sexual behavior/attitudes and sociosexuality. D3 and psychopathy were mostly associated - unsurprisingly — with moral judgments related to MELS Deception and Harm. In contrast, sexual measures and sociosexuality, while also correlated with moral judgments of Deception and Harm, were additionally related to moral judgments in the other MELS dimensions. Our results extend those of Quintelier, Ishii, Weeden, Kurzban, and Braeckman (2013) and Kurzban, Dukes, and Weeden (2010) in pointing to a relation between sexual behavior and mating orientation and morality. Our results also suggest that the association between sexual behavior and sociosexuality with moral judgments can be stronger than some personality variables. Tables 2 and 3 reveal that 11 of $30(36.7 \%)$ possible associations between sexuality and SOI-R Behavior measures with the six MELS dimensions were stronger for men than for women; only 4 of $30(13.3 \%)$ were stronger for women than men. In contrast, 5 of 12 (41.7\%) of the correlations between SOI-R Attitude and Desire measures with the MELS dimensions were stronger for women than for men, and only 2 of $12(16.7 \%)$ were stronger for men. Six of $42(14.3 \%)$ of the associations between D3 and TriPM measures with MELS dimensions were stronger for men than for women, while only 1 of $42(2.4 \%)$ was stronger for women. This pattern of associations between SOI Attitude and Desire measures and decreased moral condemnation with respect to MELS Disgust and Body dimensions suggests that more unrestricted sociosexuality manifests somewhat differently in males and females. The influence of unrestricted sociosexuality seems to parallel that of D3 traits and psychopathy, facilitating a 
selective openness to additional partners in women. These results again suggest that gender needs to be taken into account when looking at relations among personality, sexuality, and moral judgments.

\section{Conclusion}

We found that D3 components and TriPM dimensions of psychopathy were correlated with sexual behavior and attitudes, sociosexuality, and intentions toward infidelity. The pattern of relations differed for men and women, with D3 and TriPM measures generally more strongly associated with sexual behaviors in men, but more strongly with attitudinal components of sociosexuality and intention toward infidelity in women. The pattern of relations suggests that D3 traits and psychopathy may influence sexuality in ways that are linked to gender-by tending to facilitate increased numbers of matings in men, and increasing openness to focused extra-pair mating in women. Sexual behavior/attitudes and sociosexuality were strongly associated with everyday moral judgments, and—surprisingly—were generally more strongly linked to moral judgments than were D3 and TriPM measures. The present results point to the importance of gender in consideration of the roles of personality traits in human mating behaviors and moral judgments. 


\section{References}

Buss, D. M., \& Schmitt, D. P. (1993). Sexual strategies theory: An evolutionary perspective on human mating. Psychological Review, 100(2), 204-232.

Carter, G. L., Campbell, A. C., \& Muncer, S. (2014a). The dark triad personality: Attractiveness to women. Personality and Individual Differences, 56, 57-61.

Carter, G. L., Campbell, A. C., \& Muncer, S. (2014b). The Dark Triad: Beyond a 'male'mating strategy. Personality and Individual Differences, 56, 159-164.

Efferson, L. M., \& Glenn, A. L. (2018). Examining gender differences in the correlates of psychopathy: A systematic review of emotional, cognitive, and morality-related constructs. Aggression and Violent Behavior, 41, 48-61.

Ermer, E., \& Kiehl, K. A. (2010). Psychopaths are impaired in social exchange and precautionary reasoning. Psychological Science, 21(10), 1399-1405.

Greiling, H., \& Buss, D. M. (2000). Women's sexual strategies: The hidden dimension of extrapair mating. Personality and Individual Differences, 28(5), 929-963.

Hall, J. R., \& Benning, S. D. (2006). The "Successful" Psychopath: Adaptive and Subclinical Manifestations of Psychopathy in the General Population. In C. J. Patrick (Ed.), Handbook of psychopathy (pp. 459-478). New York, NY, US: The Guilford Press.

Hare, R. D. (1999). Without conscience: The disturbing world of the psychopaths among us. Guilford Press.

Holtzman, N. S., \& Strube, M. J. (2013). People with dark personalities tend to create a physically attractive veneer. Social Psychological and Personality Science, 4(4), 461-467. 
Jonason, P. K., Li, N. P., Webster, G. D., \& Schmitt, D. P. (2009). The dark triad: Facilitating a short-term mating strategy in men. European Journal of Personality: Published for the European Association of Personality Psychology, 23(1), 5-18.

Jonason, P. K., Strosser, G. L., Kroll, C. H., Duineveld, J. J., \& Baruffi, S. A. (2015). Valuing myself over others: The Dark Triad traits and moral and social values. Personality and Individual Differences, 81, 102-106.

Jones, D. N., Olderbak, \& S. G., Figueredo. (2011). The intentions towards infidelity scale. Handbook of Sexuality Related Measures, 7477.

Jones, D. N., \& Paulhus, D. L. (2014). Introducing the short dark triad (SD3) a brief measure of dark personality traits. Assessment, 21(1), 2841.

Karandikar, S., Kapoor, H., Fernandes, S., \& Jonason, P. K. (2018). Predicting moral decisionmaking with dark personalities and moral values. Personality and Individual Differences, accepted, Apr 4, 2018. Doi: 10.1016/j.paid.2018.03.048.

Kurzban, R., Dukes, A., \& Weeden, J. (2010). Sex, drugs and moral goals: Reproductive strategies and views about recreational drugs. Proceedings of the Royal Society of London B: Biological Sciences, 277(1699), 3501-3508.

Lovett, B. J., Jordan, A. H., \& Wiltermuth, S. S. (2012). Individual differences in the moralization of everyday life. Ethics \& Behavior, 22(4), 248257.

Muris, P., Merckelbach, H., Otgaar, H., \& Meijer, E. (2017). The malevolent side of human nature: A meta-analysis and critical review of the literature on the Dark Triad (Narcissism, Machiavellianism, and Psychopathy), 12, 183-204. Doi: 10.1177/1745691616666070. 
Patrick, C. J., Fowles, D. C., \& Krueger, R. F. (2009). Triarchic conceptualization of psychopathy: Developmental origins of disinhibition, boldness, and meanness. Development and Psychopathology, 21(3), 913-938.

Paulhus, D. L., \& Williams, K. M. (2002). The dark triad of personality: Narcissism, Machiavellianism, and psychopathy. Journal of Research in Personality, 36(6), 556-563.

Penke, L., \& Asendorpf, J. B. (2008). Beyond global sociosexual orientations: A more differentiated look at sociosexuality and its effects on courtship and romantic relationships. Journal of Personality and Social Psychology, 95(5), 11131135.

Quintelier, K. J., Ishii, K., Weeden, J., Kurzban, R., \& Braeckman, J. (2013). Individual differences in reproductive strategy are related to views about recreational drug use in Belgium, the Netherlands, and Japan. Human Nature, 24(2), 196-217.

Selterman, D., \& Koleva, S. (2015). Moral judgment of close relationship behaviors. Journal of Social and Personal Relationships, 32, 922-945. Doi: 10.1177/0265407514554513. 


\section{Acknowledgments}

The research was supported by the J. William and Katherine Asher Fund for Psychological

Research at DePauw University. The fund played no role in the design, analysis, or writing of the report. 
Table 1. D3 and TriPM Correlations with Sexual Behavior, Sociosexuality, and Infidelity.

\begin{tabular}{|c|c|c|c|c|c|c|c|c|c|c|c|c|c|c|}
\hline & \multicolumn{2}{|c|}{ D3 MACH } & \multicolumn{2}{|c|}{$\begin{array}{c}\text { D3 } \\
\text { NARCISSISM }\end{array}$} & \multicolumn{2}{|c|}{ D3 PSYCH } & \multicolumn{2}{|c|}{ TRIPM TOT } & \multicolumn{2}{|c|}{ BOLDNESS } & \multicolumn{2}{|c|}{ MEANNESS } & \multicolumn{2}{|c|}{ DISINHIBITION } \\
\hline & $\mathrm{M}$ & $\mathrm{F}$ & M & $\mathrm{F}$ & M & $\mathrm{F}$ & M & $\mathrm{F}$ & M & $\mathrm{F}$ & M & $\mathrm{F}$ & M & $\mathrm{F}$ \\
\hline $\begin{array}{c}\text { \# Ideal } \\
\text { Partners }\end{array}$ & .14 & -.05 & $.21^{* 2}$ & $.07^{2}$ & $.25^{* * 2}$ & $.02^{2}$ & $.31^{* * 1,2}$ & $.08^{1,2}$ & .06 & .09 & $.34^{* * 1,2}$ & $-.04^{1,2}$ & $.17^{*}$ & .11 \\
\hline $\begin{array}{c}\text { \# Past } \\
\text { Partners }\end{array}$ & $.20^{*}$ & .09 & $.29^{* * 1,2}$ & $.02^{1,2}$ & $.23^{* * 2}$ & $.08^{2}$ & $.34^{* * 2}$ & $.19^{* 2}$ & $.18^{*}$ & .14 & $.25^{* * 2}$ & $.08^{2}$ & .09 & .16 \\
\hline $\begin{array}{c}\text { Age at } 1 \text { st } \\
\text { Sex }\end{array}$ & -.01 & .002 & -0.12 & .11 & $-.19^{*}$ & -.07 & $-.19^{*}$ & -.02 & -.11 & .003 & -.11 & .12 & -.12 & -.07 \\
\hline $\begin{array}{l}\text { Multiple - } \\
\text { Single Pref }\end{array}$ & -.11 & -.04 & -.14 & -.11 & $-.38^{* * 1,2}$ & $-.14^{1,2}$ & $-.24^{* *}$ & $-.31^{* *}$ & $.02^{2}$ & $-.25^{* * 2}$ & $-.25^{* *}$ & -.15 & $-.27^{* *}$ & -.11 \\
\hline $\begin{array}{c}\text { SOI } \\
\text { BEHAV }\end{array}$ & .17 & .12 & $.36^{* * 1,2}$ & $.10^{1,2}$ & $.43^{* *_{2}}$ & $.22^{* 2}$ & $.45^{* *}$ & $.35^{* *}$ & $.30^{* *}$ & $.25^{* *}$ & $.24^{* *}$ & .11 & $.23^{* *}$ & $.36^{* *}$ \\
\hline $\begin{array}{c}\text { SOI } \\
\text { ATTITU }\end{array}$ & .16 & $.24^{* *}$ & .09 & $.21^{*}$ & $.33^{* *}$ & $.32^{* *}$ & $.21^{*}$ & $.39^{* *}$ & .05 & $.20^{*}$ & $.18^{*}$ & $.32^{* *}$ & .10 & $.27^{* *}$ \\
\hline $\begin{array}{c}\text { SOI } \\
\text { DESIRE }\end{array}$ & $.25^{* *}$ & $.29^{* *}$ & -.02 & $.18^{*}$ & $.27^{* *}$ & $.35^{* *}$ & .15 & $.37^{* *}$ & -.12 & .02 & $.20^{*}$ & $.35^{* *}$ & $.20^{*}$ & $.37^{* *}$ \\
\hline SOI TOT & $.24^{* *}$ & $.27^{* *}$ & .17 & $.21^{*}$ & $.42^{* *}$ & $.38^{* *}$ & $.33^{* *}$ & $.45^{* *}$ & .08 & $.20^{*}$ & $.23^{* *}$ & $.34^{* *}$ & $.21^{*}$ & $.40^{* *}$ \\
\hline Infidelity & $.24^{* * 2}$ & $.44^{* *_{2}}$ & $.21^{*}$ & $.30^{* *}$ & $.51^{* *}$ & $.60^{* *}$ & $.35^{* * 1}$ & $.57^{* * 1}$ & -.06 & .10 & $.42^{* * 2}$ & $.56^{* * 2}$ & $.37^{* * 1,2}$ & $.56^{* * 1,2}$ \\
\hline $\begin{array}{l}*_{--} p<0.01 \\
*_{--. p}<0.05\end{array}$ & $\begin{array}{l}\text { ailed } \\
\text { iled) }\end{array}$ & & & & & $\begin{array}{l}1--\mathrm{cc} \\
2-\mathrm{g}\end{array}$ & od & relat & ndic & by $\mathrm{s}$ & icant & actio & $<.05$ & iled) \\
\hline
\end{tabular}


Table 2. Correlations for Sexual Behavior/Attitudes, SOI, with Moral Judgments (MELS)

\begin{tabular}{|c|c|c|c|c|c|c|c|c|c|c|c|c|c|c|c|c|c|c|}
\hline & \multicolumn{2}{|c|}{$\begin{array}{c}\text { \#IDEAL } \\
\text { PARTNERS }\end{array}$} & \multicolumn{2}{|c|}{$\begin{array}{c}\text { PAST } \\
\text { PARTNERS }\end{array}$} & \multicolumn{2}{|c|}{$\begin{array}{l}\text { AGE AT } \\
1 \text { st SEX }\end{array}$} & \multicolumn{2}{|c|}{$\begin{array}{l}\text { Multiple - } \\
\text { Single Pref }\end{array}$} & \multicolumn{2}{|c|}{$\begin{array}{c}\text { SOI } \\
\text { BEHAV }\end{array}$} & \multicolumn{2}{|c|}{$\begin{array}{c}\text { SOI } \\
\text { ATTITUDE }\end{array}$} & \multicolumn{2}{|c|}{$\begin{array}{c}\text { SOI } \\
\text { DESIRE }\end{array}$} & \multicolumn{2}{|c|}{$\begin{array}{l}\text { SOI } \\
\text { TOT }\end{array}$} & \multicolumn{2}{|c|}{ INFID } \\
\hline & $\mathrm{M}$ & $\mathrm{F}$ & $\mathrm{M}$ & $\mathrm{F}$ & M & $\mathrm{F}$ & $\mathrm{M}$ & $\mathrm{F}$ & $\mathrm{M}$ & $\mathrm{F}$ & M & $\mathrm{F}$ & $\mathrm{M}$ & $\mathrm{F}$ & M & F & $\mathrm{M}$ & $\mathrm{F}$ \\
\hline Deception & $-.32^{* *}$ & -.1 & $-.20^{*}$ & -.02 & .13 & -.05 & $.22^{* *}$ & .15 & -.16 & $-.22^{*}$ & -.15 & $-.26^{* *}$ & $-.18^{*}$ & $.33^{* *}$ & $.20^{*_{2}}$ & $.35^{-* 22}$ & $.33^{* *}$ & $.22^{-}$ \\
\hline Harm & $-.23^{* * 1}$ & $.05^{1}$ & $-.29^{* *}$ & .01 & $.26^{* * 1,2}$ &.$\overline{-} .10^{1,2}$ & $.22^{* *}$ & .09 & $.22^{* *}$ & -.11 & -.16 & -.11 & -.13 & $.23^{-}$ & $-.21^{*}$ & $-.19^{*}$ & $.33^{* *}$ & $.24^{-}$ \\
\hline Lazy & $-.04^{2}$ & $-.19^{* 2}$ & -.06 & -.13 & $.22^{{ }^{1,2}}$ & $.05^{1,2}$ & -.12 & .04 & -.04 & -.15 & $-.23^{* *}$ & $-.24^{* *}$ & $.22^{-}$ & -.10 & $-.22^{*}$ & $-.22^{*}$ & $.18^{*}$ & $.20^{*}$ \\
\hline Failure & -.12 & -.16 & -.09 & -.12 & $.20^{*_{1,2}}$ & $.06^{-}$ & -.09 & -.07 & -.06 & -.12 & $-.18^{*}$ & $-.28^{* *}$ & $-.17^{*}$ & -.12 & $-.18^{*}$ & $.24^{-}$ & .07 & -.00 \\
\hline Body & $-.06^{2}$ & $-.26^{* * 2}$ & -.14 & -.16 & $.36^{* *}$ & $.18^{*}$ & .06 & .11 & $-.19^{*}$ & $.26^{-}$ & $-.49^{* *}$ & $-.52^{* *}$ & $.39^{-}$ & $.30^{-}$ & $.46^{-}$ & $.49^{* *}$ & .07 & .01 \\
\hline Disgust & $-.08^{2}$ & $-.24^{* * 2}$ & -.11 & .04 & $.26^{* *_{1,2}}$ & $.05^{1,2}$ & -.03 & .08 & .03 & -.12 & $-.12^{1,2}$ & $-.39^{* * 1,2}$ & $-.20^{*}$ & $.26^{* *}$ & $-.13^{2}$ & $.34^{-}$ & .06 & -.06 \\
\hline $\begin{array}{l}\text { MELS } \\
\text { Total }\end{array}$ & $-.19^{*_{2}}$ & $-.21^{*_{2}}$ & $-.21^{*}$ & -.08 & $.34^{* * 1,2}$ & $.01^{1,2}$ & .06 & .11 & $-.18^{*}$ & $-.21^{*}$ & $-.35^{* *}$ & $-.41^{* *}$ & $.32^{-}$ & $.31^{* *}$ & $.37^{-}$ & $.41^{* *}$ & -.09 & -.07 \\
\hline
\end{tabular}

$* *_{--} p<0.01$ (2-tailed) 1-- correlations differ by sex at .05 level (2-tailed)

$*_{--} p<0.05$ (2-tailed) $\quad 2$ - gender moderates relation, indicated by significant interaction, $\mathrm{p}<.05$ (2-tailed) 
Table 3. Correlations for D3 and TriPM with Moral Judgments (MELS)

\begin{tabular}{|c|c|c|c|c|c|c|c|c|c|c|c|c|c|c|}
\hline & \multicolumn{2}{|c|}{ D3 MACH } & \multicolumn{2}{|c|}{ D3 NARCISS } & \multicolumn{2}{|c|}{ D3 PSYCHO } & \multicolumn{2}{|c|}{ TRIPM TOT } & \multicolumn{2}{|c|}{ BOLDNESS } & \multicolumn{2}{|c|}{ MEANNESS } & \multicolumn{2}{|c|}{ DISINHIBITION } \\
\hline & M & $\mathrm{F}$ & M & $\mathrm{F}$ & M & $\mathrm{F}$ & M & $\mathrm{F}$ & M & $\mathrm{F}$ & $\mathrm{M}$ & $\mathrm{F}$ & $\mathrm{M}$ & $\mathrm{F}$ \\
\hline Deception & $-.34^{* *}$ & $-.32^{* *}$ & $-.22^{* *}$ & $-.32^{* *}$ & $-.35^{* *}$ & $-.34^{* *}$ & $-.33^{* *}$ & $-.32^{* *}$ & .02 & -.08 & $-.42^{* *}$ & $-.31^{* *}$ & $-.29^{* *}$ & $-.28^{* *}$ \\
\hline Harm & .05 & -.13 & -.11 & -.14 & $-.17^{*}$ & $-.28^{* *}$ & $-.25^{* *}$ & $-.29^{* *}$ & .11 & -.11 & $-.32^{* *}$ & $-.24^{* *}$ & $-.33^{* *}$ & $-.24^{* *}$ \\
\hline Lazy & .01 & .09 & $.23^{* *}$ & $.28^{* *}$ & $.25^{* *}$ & $.27^{* *}$ & $.34^{* *}$ & $.25^{* *}$ & .08 & $.19^{*}$ & $.27^{* *}$ & $.21^{*}$ & $.34^{* * 1}$ & $.10^{1}$ \\
\hline Failure & -.02 & -.05 & .05 & -.01 & .04 & .03 & -.01 & .04 & .121 & .10 & $-.18^{*}$ & -.05 & .04 & -.001 \\
\hline Body & -.04 & -.06 & .05 & .08 & -.02 & .04 & .06 & -.01 & -.02 & -.003 & .05 & .003 & .14 & .009 \\
\hline Disgust & $.20^{*}$ & -.02 & $.22^{* *}$ & .03 & $.22^{* * 1}$ & $-.02^{1}$ & $.18^{*}$ & -.05 & .14 & .02 & .07 & -.07 & .12 & -.04 \\
\hline $\begin{array}{c}\text { MELS } \\
\text { Total }\end{array}$ & -.001 & -.11 & .06 & -.03 & .01 & -.08 & .01 & -.09 & .09 & .02 & -.10 & -.10 & .05 & -.09 \\
\hline
\end{tabular}


